\title{
Improving Performance of WSN based Crop Monitoring System with the Help of ANN
}

\author{
Mahesh Vhatkar \\ University of Pune, Department of Computer \\ Engineering, K.J College of Engineering and \\ Management Research \\ Pune, India
}

\author{
Deepak Mehetre \\ University of Pune, Department of Computer \\ Engineering, K.J College of Engineering and \\ Management Research \\ Pune, India
}

\begin{abstract}
The main aim of this paper is to propose improvement in power of wireless sensor network technology for agricultural banana crop monitoring purpose with the help of combination of self-learning and decision making power of Artificial Neural Network and WSN, which is useful to reduce hard work of farmer's. In this work, we can use the sensor nodes with sensors attached with it such as leaf wetness, temperature sensor, soil PH, soil moisture, atmospheric pressure sensors attached to it. The values of sensors are transferred to the remote data center, at remote data center we use Artificial Neural Network, so with the help of ANN system will take better decisions and finally this better information is transferred to the end user using GSM modem. Every time transformed information is better than previous one because our ANN learns with the help of feedback from the user, according to this information system will take proper decisions for better development of the crops. The system will learn and take better decisions with new experience and feedback.
\end{abstract}

\section{General Terms}

We can classify this material under the work related to Wireless Sensor Network and Artificial Neural Network

\section{Keywords}

Wireless Sensor Network, Artificial Neural Network, Arduino Uno, Sensor Nodes, Clustering.

\section{INTRODUCTION}

All For better growth of banana plant we need better soil, required water supply and required amount of fertilizers. We should keep gap between two water supplies to this plant because soil should be dry before next water supply [1]. According to Yuan et al. US agriculture department reported that the national financial cost for agriculture rises continuously and it is for $75 \%$ of the gross income of agriculture. Traditional crop monitoring uses machines that are very costly, so it is important to reduce this cost with the help of cheap sensor nodes and WSN technology [2].Proposed work is very useful to achieve above requirements. According to Nian Mei et al. there is need of field monitoring system that can provide important data such as current condition of land, water required and other related information. This system should provide data processing platform for analysis and interpretation in the entire crop growth process. This is possible with WSN and remote sensing. [3].

According to S.Vijayakumar et al. we can use WSN for information gathering and processing in large distributed geographical area. It is very useful in monitoring in environments where manual data collection and information processing is not possible or very difficult.WSN in agriculture helps in distributed information collection and monitoring of harsh environments, we can achieve precise irrigation and better fertilizer supply to improve crop production with minimum efforts and minimum cost [5]. According to Chen $\mathrm{XianYi}$ et al. Wireless sensor network monitoring systems is very useful in poor conditions where manual work is not possible with small power consumption, rapid deployment and strong expansion i.e. we can expand our system [6]. This proposed work explains how to improve power of existing crop monitoring systems by adding power of Artificial Neural Network. Previous WSN crop monitoring systems transfers information collected from WSN directly to the farmer but in case of proposed work collected information from WSN is not transferred directly to the farmer instead it will be input to the ANN and output of ANN is the final out put which will be transferred to the end user.

\section{RELATED WORK}

Important related work is proposed by Yuan et al. Work is Calculating size of crop leaves with light intensity readings with the help of sensor readings. This system is very useful for long term monitoring with minimum number of sensor nodes and with minimum cost [2].According to work proposed by Nian Mei et al the Monitoring area is divided in to two layers, the bottom layer and upper layer. The bottom layer is made up of normal nodes and cluster headers ,normal nodes collects all the required information from the soil and sends it to the cluster heads by one hope. In upper layer there are cluster heads and sink node they are used for actual transfer of collected information from the sensors. Cluster heads sends their information to sink by multihope .In that work normal nodes are arranged to form triangle to reduce number of nodes required. Clustering is very useful for load balancing and high availability [3]. Another most important work is written by SonalVerma et al. is use of WSN to detect fire hazard, this system also used EEPROM to store sensor data in case of power failure. One example of how crop management can be enhanced using WSN is the project Accenture carried out in Northern California. Accenture Technology Labs installed a WSN at Pickberry Vineyard across a 30-acre area to continually sense physical parameters such as air temperature. Here data from sensors is combined with data collected from other source since viticulture is highly sensitive to environmental changes so the sensor data is mixed with other information collected which is better for crop growth. One more application of Wireless sensor network in agriculture field is Greenhouse monitoring [4].

According to work by S.Vijayakumar et al. India being an agricultural country needs some new advanced technology in 
the field of agriculture. It is possible with the devices having computing, communicating and controlling capability.WSN is used to control water sprinkler during the period of water scarcity. The working of water sprinkler is controlled by WSN to conserve water. They have also used soil $\mathrm{pH}$ sensors to inform to the farmers about current $\mathrm{pH}$ of soil so that farmer can use proper fertilizers. Mostly farmer needs manual data collection and it is restricted to shallow soil layers, less accurate and not suitable for real-time application [5].Another related work is by Chen XianYi et al. Here plant electrolyte power is used to implement an energy saving mechanism. This energy saving mechanism is very useful in many WSN applications which are related to the plants [6].

\section{POWER OF ANN}

Artificial neural network is very similar to the Biological neural network but not exactly same as biological neural network because our biological neural network is very complicated and works parallel with other elements of network. It is very difficult to construct exactly similar network. The building block of artificial neural network is Neuron, it is called as perceptron .Each neuron has one or more input values, each input connection is provided with some weight .Neuron calculates the weighted sum of input values, if this sum is more than or equal to threshold value then neuron will fire otherwise it will not fire .In most of neural networks 1 is used to indicate neuron is fired and 0 indicates neuron is not fired. For simple task one or some neurons are sufficient but for more complicated task we require large number of these processing elements. Number of neurons is depends on application we are going to develop.

According to b.yegnanarayana we can consider artificial neural network as a simplified model of the existing natural biological neural network. It is made up of communicating and an interconnected processing unit .The basic model is made up of two components first is summing part and next part is an output part. The first component accepts total $\mathrm{N}$ number of inputs; it multiplies each input value with the weights associated with each neuron and finally performs the weighted addition [7]. With the help of artificial neural network we can create systems which can work similar to human brain. In proposed work we can use neural network to give better information about crop development to the user, first we can train neural network by using initial sensor values. When neural network is working it can take decisions by using feedback from the user and new sensor values.

\section{HARDWARE REQUIRED}

\subsection{Arduino Uno}

Figure 1 shows [8] Arduino Uno it is a readymade circuit platform which is very useful as base for microcontroller, this microcontroller is a mini computer it is provided with processing and storing capacity, there is also provision for input and output. We can use ATmega328 for working with Arduino Uno We can say that Arduino Uno can provide required resources to the microcontroller. We can give power supply to the board by using external power supply such as external battery or with the help of USB connecter. This board may work for minimum $5 \mathrm{v}$ but for better performance and stability voltage should be 9 or 10 v. ATmega328 microcontroller works with flash memory, this flash memory is used for program storage .14 digital I/O pins and 6 analogue pins are provided for $\mathrm{I} / \mathrm{O}$ purpose, this device can work with computer or we can use it without computer for other applications such as wireless sensor networks. Arduino Uno is very useful to monitor environment around it with the help of sensors attached to it. By using physical parameters collected by sensors we can control various devices automatically, but application of this device is not limited to this area. Figure 2 shows example of an Aurduino compatible Soil moisture sensor [11].

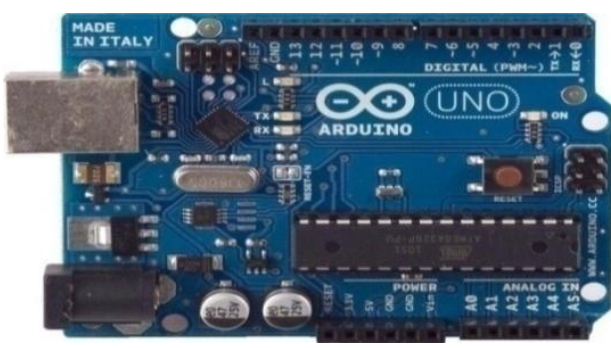

Fig 1:Arduino Uno

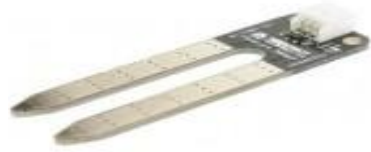

Fig 2: Soil Moisture Sensor

\subsection{Rocket M2 base station}

Figure 3 shows M2 base station[10] This is new advanced type of base station The data transmission rang of $\mathrm{m} 2$ base station is very high, it can communicate over range of 50 kilometer this is very useful for large distributed applications. It can be used with many types of antennas used today such as roof antenna and wall antenna. Data transmission range may be above $150 \mathrm{Mbps}$ for TCP/IP .We will use this base station for proposed work.

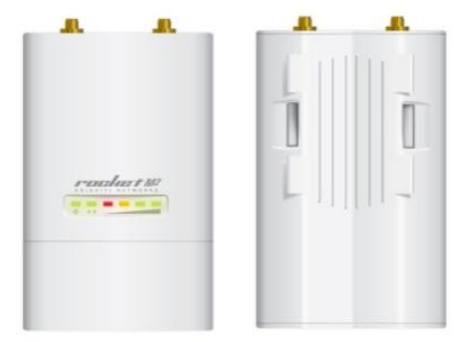

Fig 3: Rocket M2 Base Station

\section{SOFTWARE REQUIRED}

\subsection{PHP}

PHP is very useful for providing GUI based programming environment. PHP is very useful for creating dynamic websites; it is not possible with traditional HTML pages. The advantage of dynamic website is the content of website may change by user action. It is also possible to create static web pages with PHP we can also work with database while using dynamic language such as PHP. We can say that PHP is server side scripting language, server side means our webpage is created at server side and with the help of generated HTML web browser displays the related page. The syntax is very similar to ' $\mathrm{C}$ ' language, so programmers who are familiar with $\mathrm{C}$ language can easily work with PHP .It is very useful to 
work with MYSQL but we can also use it with other data base like oracle .Web browser sends the HTTP request with the help of URL (Uniform resource locator) to the web server, the web server locates the corresponding PHP pages and Interprets it to HTML code. We are using PHP for our proposed work because PHP is an open source and platform independent.

\subsection{MySQL}

It is DBMS used to manage large relational data .This is completely open source so we can make changes to it according to our requirement and because of this it is highly popular for database management. In proposed work we want database for storing collected information and some processed information from the sensor nodes .In this work we are going to use PHP for graphical user interface and MySQL can well work with PHP.one of the reason for using MySQL for proposed work because it is very fast i.e. data processing speed of MYSQL is higher than other database management systems and we can use this DBMS for any type of platforms. Source code is also available to the user.

\subsection{Matlab}

MATLAB is very useful for generation of complicated algorithms and for visualization purpose. It is also very useful for performing complex numerical calculations. We can also perform vector operations, matrices operations and simulation with the help of MATLAB. We will use the software for our neural network related work.

\section{TECHNOLOGICAL FRAMEWORK}

\subsection{Clustered monitoring system}

According to Jun Zheng et al. clustering is energy - efficient communication protocol that can be used by the sensors to report their sensed data to the sink [9]. It is very useful to organize sensor nodes hierarchically, to form cluster of nodes, where the cluster members send their data to the cluster heads while the cluster heads performs the task of transmission of information towards the sink. It is useful to give task of parameter sensing to the node having lower energy and send the sensed data to its cluster head at short distance, while a node with higher energy can be selected as a cluster head to process the data from its cluster members and transmit the processed data to the sink [9]. This process can not only reduce the energy consumption for communication, but also balance traffic load and improve scalability when the network size grows. Since all sensor nodes transmits the information with same capacity so to balance the traffic clustering should performed time to time. Data aggregation can be performed at cluster heads to reduce the amount of data transmitted to the sink and improves the energy efficiency of the network. The major problem with clustering is how to select the cluster heads and how to organize the clusters [9].

There are many cluster formation methods. With the help of distance between the members and their cluster heads, it is possible to create sensor network into a single - hop clustering architecture or a multihop clustering architecture, as shown in Figs. 4 and 5, respectively [9].

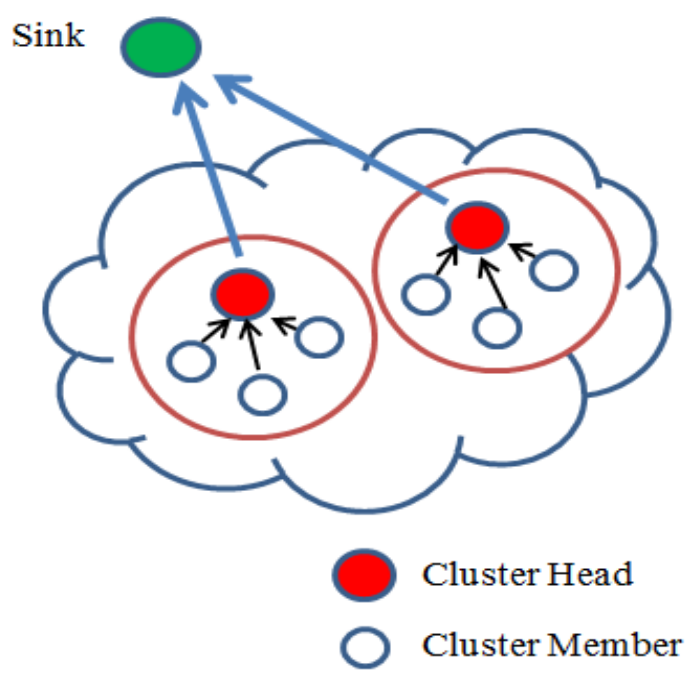

Fig 4: Single Hop Clustering Architecture

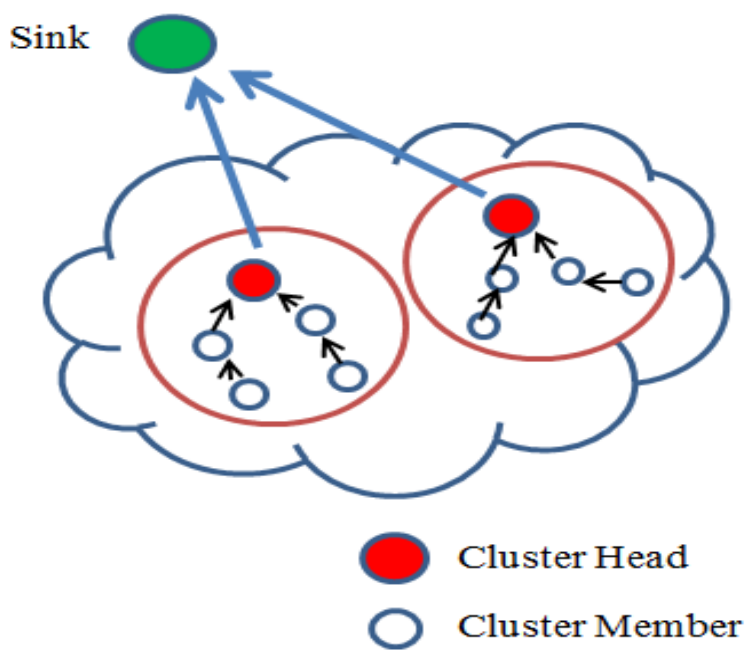

Fig 5: Multi Hop Clustering Architecture

It is very useful to divide area under monitoring in to the clusters .According to work by Nian Mei et al. Area is divided into two layers, lower layer and upper layer, lower layer consist of normal nodes and cluster heads. This lower layer collects all the information collected by sensors .Upper layer is useful for transmission purpose, it is consists of cluster headers and sink .Sink transfers data to the base station [3].This idea is very useful for proposed work also.

\subsection{Working principle and application of ANN at remote station}




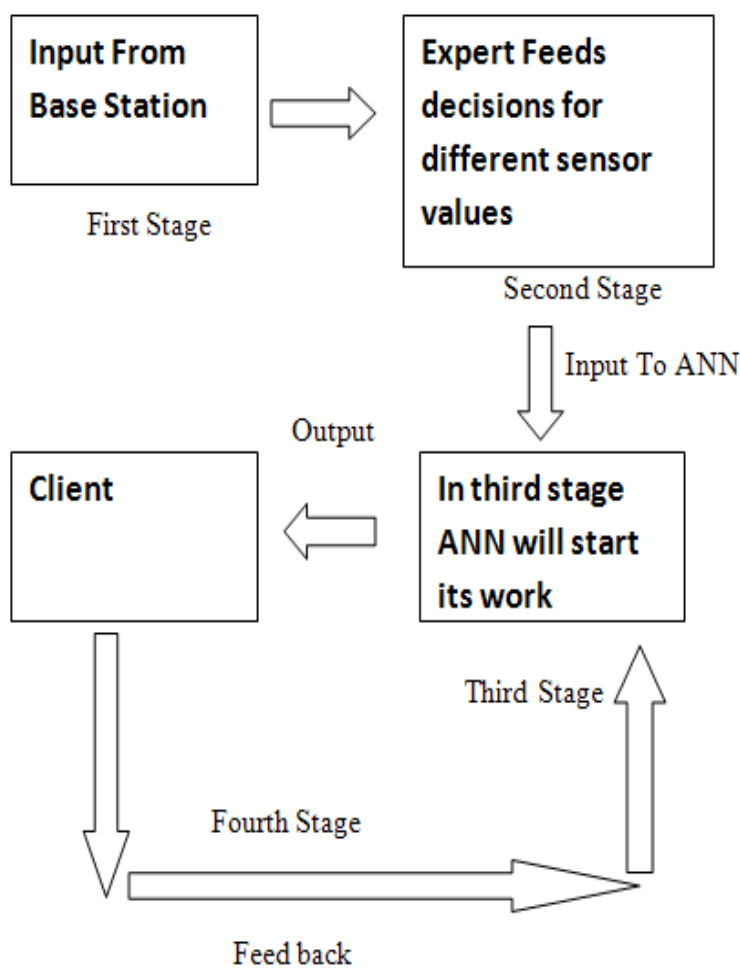

Fig 6: Working Principle

Fig 6 shows the block diagram of proposed system. This work is divided into four stages, as shown in figure 6.In the first stage base station will send its collected information to the remote center, at remote center we have ANN software we have to train it. Now we need agricultural exports. Now in the second stage agricultural expert will read sensor values coming from the base station and he will feed corrective actions that should be taken for different sensor values, these corrective actions may include which fertilizers should be better for the current agricultural condition of the farm, which crop farmer should take, if there is any need of water supply to the farm and it may include many other corrective actions. This process will be continue for the three month, after three months in the third stage the system ANN will take decisions by its own because ANN is trained with the help of agricultural expert and current sensor values. Now there is no need of any agricultural export because our system is now working with the help of ANN.

Finally in the fourth stage the feedback taken from the user about the work is act as input to the ANN so system learns every time and will take better decisions with the help of new feedback. Fig 07 shows system under training and fig 08 shows working system.

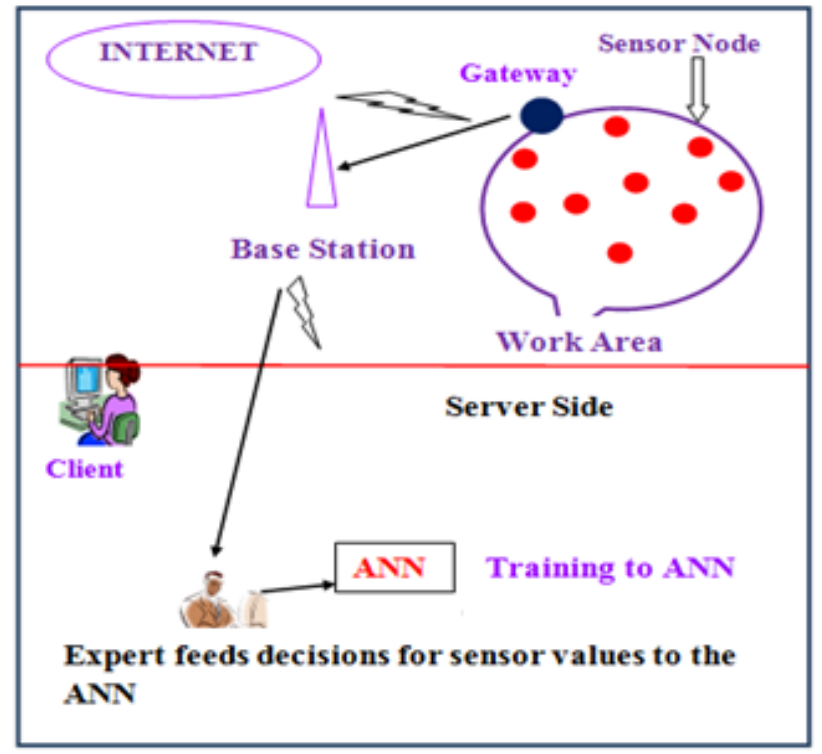

Fig 7: System under training.

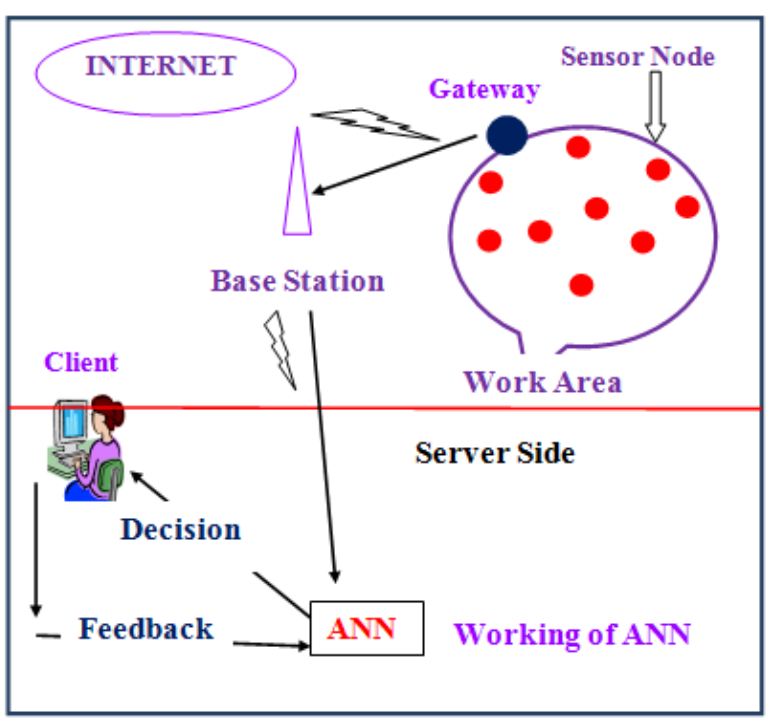

Fig 8: Working system.

\section{CONCLUSION}

This work is very useful for agriculture purpose to solve many problems related to banana plant growth; this work adds benefits of ANN to the existing wireless sensor network technology, to give better information related to the farm and crop devilment to the farmer.

\section{REFRENCES}

[1] http://www.banana-tree.com

[2] Yuan Yuan, Shanshan Li, KuiWu, WeijiaJia and YuxingPeng, "FOCUS: A Cost-Effective Approach for Large-Scale Crop Monitoring with Sensor Networks", Mobile Adhoc and Sensor Systems, MASS '09. IEEE 6th International Conference, Oct 2009.

[3] Nian Mei, Yiping YUAN, "A Technical Framework for Designing Wireless Sensor Networks for Agricultural Monitoring in XinJiang Regions”, Education Technology 
and Computer Science (ETCS), Second International Workshop on Date of Conference: 6-7 March 2010.

[4] SonalVerma, Nikhil Chug, Dhananjay V. Gadre ,Wireless Sensor Network for Crop Field Monitoring", International Conference on Recent Trends in Information Telecommunication and Computing Kochi , 12-13 March 2010.

[5] s. Vijayakumar, j. Nelson rosario, "Preliminary design for crop monitoring involving water and fertilizer conservation using wireless sensor networks" ,IEEE 3rd international conference, date 27-29 may 2011.

[6] Chen XianYi , Jin ZhiGang, YangXiong, "Design of Tropical crops pests monitoring system based on wireless sensor network" , Consumer Electronics,
Communications and Networks (CECNet), 2012 2nd International Conference, Date 21-23 April 2012.

[7] B.yegnanarayana, Artificial Neural Networks, published by Asoke K Ghosh, PHI Learning Private Limited, Press Rajkamal Electronic, January 2012

[8] http://www.arduino.cc

[9] Jun Zheng , Abbas Jamalipour, Wireless Sensor Networks a Networking Perspective, IEEE Press 445 Hoes Lane Piscataway, 2009

[10] http://www.ubnt.com/marketing.

[11] http://www.dfrobot.com 